\title{
Sortable simplicial complexes and $t$-independence ideals of proper interval graphs
}

\author{
Jürgen Herzog \\ Fachbereich Mathematik \\ Universität Duisburg-Essen, Campus Essen \\ 45117 Essen, Germany \\ juergen.herzog@uni-essen.de \\ Fahimeh Khosh-Ahang Somayeh Moradi \\ Department of Mathematics, School of Science \\ Ilam University \\ P.O.Box 69315-516, Ilam, Iran \\ $\{$ f.khoshahang, so.moradi\}@ilam.ac.ir \\ Masoomeh Rahimbeigi \\ Department of Mathematics \\ University of Kurdistan \\ Post Code 66177-15175, Sanandaj, Iran \\ rahimbeigi_masoome@yahoo.com
}

Submitted: Jul 8, 2019; Accepted: Mar 5, 2020; Published: Mar 20, 2020

(C) The authors. Released under the CC BY-ND license (International 4.0).

\begin{abstract}
We introduce the notion of sortability and $t$-sortability for a simplicial complex and study the graphs for which their independence complexes are either sortable or $t$-sortable. We show that the proper interval graphs are precisely the graphs whose independence complex is sortable. By using this characterization, we show that the ideal generated by all squarefree monomials corresponding to independent sets of vertices of $G$ of size $t$ (for a given positive integer $t$ ) has the strong persistence property, when $G$ is a proper interval graph. Moreover, all of its powers have linear quotients.
\end{abstract}

Mathematics Subject Classifications: 13F20, 05E45 


\section{Introduction}

The notion of strong persistence property for an ideal in a Noetherian ring $R$ has been defined in [8]. It is known that any monomial ideal with the strong persistence property has the persistence property (see [8]). Although finding ideals with the strong persistence property is of great interest, but there is not much known about them. Few classes of monomial ideals are known to possess this property. Polymatroidal ideals ([9]) and edge ideals of graphs ([12]) are some of these families. In this paper, we introduce a new class of monomial ideals associated to proper interval graphs with the strong persistence property. To this aim, we introduce the notion of a sortable simplicial complex and show that the independence complex of a graph $G$ is sortable if and only if $G$ is a proper interval graph. Using this characterization, we obtain some algebraic properties of the $t$-independence ideal $I_{t}(G)$ generated by all squarefree monomials corresponding to independent sets of vertices of $G$ of size $t$, when $G$ is a proper interval graph. It is proved that this ideal has the strong persistence property. Moreover, when $G$ is a proper interval graph or an $n$-cycle, it is shown that the toric ring $K\left[u: u \in \mathcal{G}\left(I_{t}(G)\right)\right]$ over the field $K$ is Koszul and a normal Cohen-Macaulay domain.

We recall some definitions and notation that are needed in the sequel. Let $G$ be any finite simple graph on the vertex set $V$. A subset $F \subseteq V$ is called an independent set of $G$ if it contains no edge of $G$. The set of all independent sets of $G$ forms a simplicial complex $\Delta(G)$, which is called the independence complex of $G$. For a graph $G$ on the vertex set $[n]$, a subset $A \subseteq[n]$ is called an interval in $G$, if $A=\{r, r+1, \ldots, s\}$ for some $r \leqslant s$. The set of all vertices adjacent to a vertex $v$ in $G$ is denoted by $N_{G}(v)$ and by $N_{G}[v]$ we mean $N_{G}(v) \cup\{v\}$. The path graph and the cycle graph with $n$ vertices are denoted by $P_{n}$ and $C_{n}$, respectively.

A graph $G$ is called an interval graph, if one can label its vertices with some intervals on the real line so that two vertices are adjacent in $G$, when the intersection of their corresponding intervals is non-empty. A proper interval graph is an interval graph such that no interval properly contains another. Proper interval graphs are well studied in the literature, see for example $[1,4,11,15]$. In this paper we give another characterization of these graphs in terms of the sortability of their independence complexes.

Let $S=K\left[x_{1}, \ldots, x_{n}\right]$ be a polynomial ring over a field $K$ and $u$ and $v$ be two monomials of degree $d$ in $S$. Write $u v=x_{i_{1}} x_{i_{2}} \cdots x_{i_{2 d}}$ with $1 \leqslant i_{1} \leqslant i_{2} \leqslant \cdots \leqslant i_{2 d}$, and set $u^{\prime}=x_{i_{1}} x_{i_{3}} \cdots x_{i_{2 d-1}}$ and $v^{\prime}=x_{i_{2}} x_{i_{4}} \cdots x_{i_{2 d}}$. The pair $\left(u^{\prime}, v^{\prime}\right)$ is called the sorting of $(u, v)$ and is denoted by $\operatorname{sort}(u, v)$. Note that if $u$ and $v$ are squarefree, then $u^{\prime}$ and $v^{\prime}$ are squarefree, as well. The pair $(u, v)$ is called a sorted pair, if $\operatorname{sort}(u, v)=(u, v)$. Otherwise, $(u, v)$ is called an unsorted pair. Let $S_{d}$ be the set of all monomials of degree $d$ in $S$. A subset $\mathcal{M} \subset S_{d}$ is called sortable if $\operatorname{sort}(u, v) \in \mathcal{M} \times \mathcal{M}$ for all $(u, v) \in \mathcal{M} \times \mathcal{M}$. We say that a monomial ideal $I$ is sortable, if it is generated in a single degree and $\mathcal{G}(I)$ is a sortable set of monomials, where $\mathcal{G}(I)$ is the set of minimal monomial generators of $I$.

The paper proceeds as follows. In Section 2, we introduce and study sortable and $t$-sortable simplicial complexes. As one of the main results of this section, we give a new characterization of proper interval graphs by means of sortability concept (see Theorem 
8). Moreover, we prove that the independence complex of any cycle graph is $t$-sortable. In Section 3, we consider the $t$-independence ideals of proper interval graphs and prove that these ideals satisfy the $\ell$-exchange property and consequently the strong persistence property. Finally we show that for any ideal in this class, all of its powers have linear quotients and hence linear resolutions.

\section{Sortable simplicial complexes}

Let $\Delta$ be a (finite) simplicial complex on the vertex set $V(\Delta) \subset \mathbb{N}$. For any finite set $F \subset \mathbb{N}$, we associate with $F$ the monomial $\mathbf{x}^{F}=\prod_{i \in F} x_{i}$.

Given two faces $F, G \in \Delta$ with $|F|=r$ and $|G|=s$ we write

$$
\mathbf{x}^{F} \mathbf{x}^{G}=x_{i_{1}} x_{i_{2}} \cdots x_{i_{r+s}} \quad \text { with } \quad i_{1} \leqslant i_{2} \leqslant \cdots \leqslant i_{r+s} .
$$

We define the sorting operator as follows:

$$
\operatorname{sort}(F, G)=\left(F^{\prime}, G^{\prime}\right)
$$

where $F^{\prime}=\left\{i_{k}: 1 \leqslant k \leqslant r+s, k\right.$ is odd $\}$ and $G^{\prime}=\left\{i_{k}: 1 \leqslant k \leqslant r+s, k\right.$ is even $\}$.

Notice that $\left|F^{\prime}\right|=\left|G^{\prime}\right|$ if $r+s$ is even, and $\left|F^{\prime}\right|=\left|G^{\prime}\right|+1$ if $r+s$ is odd.

Definition 1. Let $\Delta$ be a finite simplicial complex with $V(\Delta) \subset \mathbb{N}$. Then $\Delta$ is sortable with respect to the given labeling on $V(\Delta)$, if for any $F, G \in \Delta$, one has $\operatorname{sort}(F, G) \in$ $\Delta \times \Delta$. Moreover, $\Delta$ is sortable, if it is sortable with respect to some suitable labeling with integers on $V(\Delta)$.

A weaker property than sortability which is called $t$-sortability is defined as follows.

Definition 2. Let $t$ be a positive integer. A finite simplicial complex $\Delta$ with $V(\Delta) \subset \mathbb{N}$ is called $t$-sortable with respect to the given labeling on $V(\Delta)$, if for any $F, G \in \Delta$ with $|F|=|G|=t$ we have $\operatorname{sort}(F, G) \in \Delta \times \Delta$. Moreover, $\Delta$ is $t$-sortable, if it is $t$-sortable with respect to some suitable labeling with integers on $V(\Delta)$.

Note that if $\Delta$ is $t$-sortable and $F, G \in \Delta$ such that $|F|=|G|=t$ and $\operatorname{sort}(F, G)=$ $\left(F^{\prime}, G^{\prime}\right)$, then $\left|F^{\prime}\right|=\left|G^{\prime}\right|=t$.

We have the following simple observations.

Remark 3. Let $\Delta$ be a finite simplicial complex with $V(\Delta) \subset \mathbb{N}$.

(i) If $\Delta$ is sortable ( $t$-sortable), then for any $T \subset V(\Delta)$, the simplicial complex $\Delta_{T}=$ $\{F \in \Delta: F \subset T\}$ is also sortable (t-sortable).

(ii) If $\Delta$ is sortable, then it is t-sortable for any positive integer $t$.

(iii) The converse of (ii) does not hold in general. Indeed for any $n \geqslant 4, \Delta\left(C_{n}\right)$ is $t$-sortable for all $t$ and is not sortable (see Theorem 8 and Proposition 11). 
Recall that if $\Delta_{1}$ and $\Delta_{2}$ are simplicial complexes on disjoint sets of vertices, then the join of $\Delta_{1}$ and $\Delta_{2}$ denoted by $\Delta_{1} * \Delta_{2}$ is a simplicial complex on the vertex set $V\left(\Delta_{1} * \Delta_{2}\right)=V\left(\Delta_{1}\right) \cup V\left(\Delta_{2}\right)$ defined as $\Delta_{1} * \Delta_{2}=\left\{F \cup G: F \in \Delta_{1}\right.$ and $\left.G \in \Delta_{2}\right\}$.

Proposition 4. Let $\Delta_{1}$ and $\Delta_{2}$ be simplicial complexes on disjoint sets of vertices. Then $\Delta_{1} * \Delta_{2}$ is sortable, if and only if $\Delta_{1}$ and $\Delta_{2}$ are sortable.

Proof. Let $\Delta_{1}$ and $\Delta_{2}$ be sortable. One may consider sorting labelings on the vertices of $\Delta_{1}$ and $\Delta_{2}$ in $\mathbb{N}$ such that $i<j$ for all $i \in V\left(\Delta_{1}\right)$ and $j \in V\left(\Delta_{2}\right)$. Consider two elements $F_{1} \cup F_{2}$ and $G_{1} \cup G_{2}$ in $\Delta_{1} * \Delta_{2}$ with $F_{1}, G_{1} \in \Delta_{1}$ and $F_{2}, G_{2} \in \Delta_{2}$. Let $\left(F_{1}^{\prime}, G_{1}^{\prime}\right)=\operatorname{sort}\left(F_{1}, G_{1}\right)$ and $\left(F_{2}^{\prime}, G_{2}^{\prime}\right)=\operatorname{sort}\left(F_{2}, G_{2}\right)$. One can see that if $\left|F_{1}\right|+\left|G_{1}\right|$ is even, then $\operatorname{sort}\left(F_{1} \cup F_{2}, G_{1} \cup G_{2}\right)=\left(F_{1}^{\prime} \cup F_{2}^{\prime}, G_{1}^{\prime} \cup G_{2}^{\prime}\right)$ and if $\left|F_{1}\right|+\left|G_{1}\right|$ is odd, then $\operatorname{sort}\left(F_{1} \cup F_{2}, G_{1} \cup G_{2}\right)=\left(F_{1}^{\prime} \cup G_{2}^{\prime}, G_{1}^{\prime} \cup F_{2}^{\prime}\right)$. Since $F_{1}^{\prime}, G_{1}^{\prime} \in \Delta_{1}$ and $F_{2}^{\prime}, G_{2}^{\prime} \in \Delta_{2}, \Delta_{1} * \Delta_{2}$ is sortable.

Conversely, let $\Delta_{1} * \Delta_{2}$ be sortable. For any two faces $F, G \in \Delta_{1}$, since $F, G \in \Delta_{1} * \Delta_{2}$, we have $F^{\prime}, G^{\prime} \in \Delta_{1} * \Delta_{2}$, where $\left(F^{\prime}, G^{\prime}\right)=\operatorname{sort}(F, G)$. Note that $F^{\prime}, G^{\prime} \subseteq V\left(\Delta_{1}\right)$ and $V\left(\Delta_{1}\right) \cap V\left(\Delta_{2}\right)=\emptyset$. This implies that $F^{\prime}, G^{\prime} \in \Delta_{1}$. By similar argument $\Delta_{2}$ is also sortable.

Let $G$ be the disjoint union of two graphs $G_{1}$ and $G_{2}$. Then

$$
\Delta(G)=\Delta\left(G_{1}\right) * \Delta\left(G_{2}\right) .
$$

Thus, we may apply Proposition 4 and obtain

Corollary 5. Let $G$ be a finite simple graph with vertices in $\mathbb{N}$ and $G_{1}, \ldots, G_{m}$ be the connected components of $G$. Then $\Delta(G)$ is sortable, if and only if each $\Delta\left(G_{r}\right)$ is sortable.

Remark 6. If we replace sortability by t-sortability in Corollary 5, the 'only if' part holds by Remark 3(i). But the 'if' part does not hold in general. For example, consider a graph $G$ with two connected components $G_{1}$ and $G_{2}$, where $G_{1}$ is a star graph on 4 vertices and $G_{2}$ is a path graph on 4 vertices. Then by CoCoA computations one can see that the defining ideal of the fiber ring of $I_{3}(G)=\left\langle x^{F}: F \in \Delta(G),|F|=3\right\rangle$ is not quadratic. So by Theorem 12, $I_{3}(G)$ is not a sortable ideal. Hence $\Delta(G)$ is not 3-sortable. But it is easy to see that $\Delta\left(G_{1}\right)$ and $\Delta\left(G_{2}\right)$ are 3 -sortable.

The following lemma states some equivalent conditions for a graph to be proper interval. We use this result in Theorem 8 to characterize the graphs whose independence complexes are sortable.

Lemma 7. For a graph $G$ on the vertex set $[n]$, the following conditions are equivalent:

(i) For all $i<j,\{i, j\} \in E(G)$ implies that the induced subgraph of $G$ on $\{i, i+1, \ldots, j\}$ is a clique.

(ii) For all $1 \leqslant i \leqslant n, N_{G^{i}}[i]$ is both a clique and an interval, where $G^{i}$ is the induced subgraph of $G$ on $\{i, i+1, \ldots, n\}$. 
(iii) For all $1 \leqslant i \leqslant n, N_{G_{i}}[i]$ is both a clique and an interval, where $G_{i}$ is the induced subgraph of $G$ on $\{1,2, \ldots, i\}$.

(iv) For all $1 \leqslant i \leqslant n, N_{G}[i]$ is an interval.

(v) $G$ is a proper interval graph.

Proof. (i) $\Rightarrow$ (ii) Suppose that $1 \leqslant i \leqslant n$ and $j$ is the largest integer such that $j \in N_{G^{i}}[i]$. Then $\{i, j\} \in E(G)$ or $i=j$. So, by (i) the induced subgraph of $G$ on $\{i, i+1, \ldots, j\}$ is a clique. This proves the result.

(ii) $\Rightarrow$ (iii) Suppose that $1 \leqslant i \leqslant n$ and $j$ is the least integer such that $j \in N_{G_{i}}[i]$. Then $i \in N_{G^{j}}[j]$. Thus (ii) implies that the induced subgraph of $G$ on $\{j, j+1, \ldots, i\}$ is a clique. This shows that $N_{G_{i}}[i]$ is a clique and an interval as desired.

(iii) $\Rightarrow$ (iv) Suppose that $1 \leqslant i \leqslant n$ and $j, k \in N_{G}[i]$ with $j<k$. It is enough to prove that for each integer $\ell$ between $j$ and $k$, one has $\ell \in N_{G}[i]$. If $i \leqslant j<k$, then the result follows from $i \in N_{G_{k}}[k]$ and the assumption that $N_{G_{k}}[k]$ is both clique and interval. If $j<k \leqslant i$, then the result follows from $j \in N_{G_{i}}[i]$ and the assumption that $N_{G_{i}}[i]$ is an interval. Now, assume that $j \leqslant i \leqslant k$. then $i \in N_{G_{k}}[k]$ and $j \in N_{G_{i}}[i]$. Since $N_{G_{k}}[k]$ is a clique and an interval and $N_{G_{i}}[i]$ is an interval, the result is obtained.

(iv) $\Rightarrow$ (i) Suppose that $i<j$ and $\{i, j\} \in E(G)$. Since $N_{G}[i]$ is an interval and $j \in N_{G}[i],\{i, \ldots, j\} \subseteq N_{G}[i]$. Now for each $i<\ell \leqslant j$, since $i \in N_{G}[\ell]$ and $N_{G}[\ell]$ is an interval, $\{i, i+1, \ldots, \ell\} \subseteq N_{G}[\ell]$. This shows that the induced subgraph of $G$ on $\{i, i+1, \ldots, j\}$ is a clique.

(i) $\Leftrightarrow(\mathrm{v})$ See [11, Theorem 1 and Proposition 1].

Property (iii) of Lemma 7, implies that any proper interval graph has a perfect elimination ordering and hence is a chordal graph.

Theorem 8. Let $G$ be a graph. Then $\Delta(G)$ is sortable if and only if $G$ is a proper interval graph.

Proof. Let $\Delta(G)$ be sortable and by contrary assume that $G$ is not proper interval. Then by Lemma 7 , for any labeling on $V(G)$ there exists $i \in V(G)$ such that $N_{G}[i]$ is not an interval. This means that there exists $j, k \in N_{G}[i]$ and an integer $\ell$ with $j<\ell<k$ such that $\ell \notin N_{G}[i]$. If $i<\ell$, then $\operatorname{sort}(\{i, \ell\},\{k\})=(\{i, k\},\{\ell\})$ and $\{i, k\} \notin \Delta(G)$, a contradiction. If $i>\ell$, then $\operatorname{sort}(\{i, \ell\},\{j\})=(\{j, i\},\{\ell\})$ and $\{j, i\} \notin \Delta(G)$, which contradicts to sortability of $\Delta(G)$.

Conversely, suppose that $G$ is a proper interval graph. Then by Lemma 7, we may consider a labeling on $V(G)=[n]$ such that for all $i<j,\{i, j\} \in E(G)$ implies that the induced subgraph of $G$ on $\{i, i+1, \ldots, j\}$ is a clique. Let $F_{1}, F_{2} \in \Delta(G)$ and assume that $x^{F_{1}} x^{F_{2}}=x_{i_{1}} x_{i_{2}} \cdots x_{i_{r+s}}$, where $i_{1} \leqslant i_{2} \leqslant \cdots \leqslant i_{r+s}$. Then $\operatorname{sort}\left(F_{1}, F_{2}\right)=\left(F_{1}^{\prime}, F_{2}^{\prime}\right)$, where $F_{1}^{\prime}=\left\{i_{1}, i_{3}, \ldots, i_{r^{\prime}}\right\}$ and $F_{2}^{\prime}=\left\{i_{2}, i_{4}, \ldots, i_{s^{\prime}}\right\}$ for some $r^{\prime}$ and $s^{\prime}$. By contradiction if $F_{1}^{\prime} \notin \Delta(G)$, then $\left\{i_{2 k-1}, i_{2 \ell-1}\right\} \in E(G)$ for some $k$ and $\ell$ with $k<\ell$. Since $i_{2 k-1} \leqslant i_{2 k} \leqslant$ $i_{2 \ell-1}$, by our assumption, $\left\{i_{2 k-1}, i_{2 k}\right\},\left\{i_{2 k}, i_{2 \ell-1}\right\} \in E(G)$. Note that at least two distinct vertices among $i_{2 k-1}, i_{2 k}, i_{2 \ell-1}$ belong to either $F_{1}$ or $F_{2}$. This implies that either $F_{1}$ or $F_{2}$ contains an edge, a contradiction. Thus $F_{1}^{\prime} \in \Delta(G)$. By similar argument $F_{2}^{\prime} \in \Delta(G)$. 
Corollary 9. Let $G$ be a tree. If $\Delta(G)$ is sortable, then $G$ is claw-free.

Proof. Let $\Delta(G)$ be sortable and suppose that $G$ contains a claw which is an induced subgraph $H$ with three edges $\{i, j\},\{i, l\}$ and $\{i, m\}$, for distinct vertices $i, j, l$ and $m$ of $G$. We show that $\Delta(H)$ is not sortable. Let $F=\{i\}$ and $K=\{j, l, m\}$. Then $\operatorname{sort}(F, K)=\left(F^{\prime}, K^{\prime}\right)$, where $\left|F^{\prime}\right|=\left|K^{\prime}\right|=2$ and one of $F^{\prime}$ and $K^{\prime}$ contains $i$. Thus we have either $F^{\prime} \notin \Delta(H)$ or $K^{\prime} \notin \Delta(H)$. So $\Delta(H)$ is not sortable. This contradicts to Remark 3(i), noting that $\Delta(H)=\Delta(G)_{V(H)}$.

By Corollaries 5 and 9 one can get the following result.

Corollary 10. Let $G$ be a forest. Then $\Delta(G)$ is sortable, if and only if each tree of the forest is a path graph.

The independence complex of an $n$-cycle for $n \geqslant 4$ is not sortable by Theorem 8 and Lemma 7. But we still have

Proposition 11. $\Delta\left(C_{n}\right)$ with the standard labeling on $C_{n}$ is $t$-sortable for all $t$.

Proof. For $n=3$, the assertion is trivial. Let $n \geqslant 4$, and let $A$ and $B$ be two $t$-independent sets of $C_{n}$ and $\operatorname{sort}(A, B)=\left(A^{\prime}, B^{\prime}\right)$. Note that $\Delta\left(C_{n}\right)=\left\{F \in \Delta\left(P_{n}\right):\{1, n\} \nsubseteq F\right\}$. So we have $A, B \in \Delta\left(P_{n}\right)$. Note that any path graph is a proper interval graph. Thus Theorem 8 implies that $A^{\prime}, B^{\prime} \in \Delta\left(P_{n}\right)$. Let $x^{A} x^{B}=x_{i_{1}} x_{i_{2}} \cdots x_{i_{2 t-1}} x_{i_{2 t}}$ with $i_{1} \leqslant i_{2} \leqslant$ $\cdots \leqslant i_{2 t}$. If $i_{1}>1$ or $i_{2 t}<n$, then $\{1, n\} \nsubseteq A^{\prime}$ and $\{1, n\} \nsubseteq B^{\prime}$. Therefore $A^{\prime}, B^{\prime} \in \Delta\left(C_{n}\right)$ and we are done. Now, let $i_{1}=1$ and $i_{2 t}=n$. Note that $1<i_{2}$ and $i_{2 t-1}<n$, otherwise $A$ or $B$ would not be an independent set. By definition $A^{\prime}=\left\{1, i_{3}, \ldots, i_{2 t-1}\right\}$ and $B^{\prime}=\left\{i_{2}, \ldots, i_{2 t-2}, n\right\}$. Thus $n \notin A^{\prime}$ and $1 \notin B^{\prime}$. Hence $\{1, n\} \nsubseteq A^{\prime}$ and $\{1, n\} \nsubseteq B^{\prime}$. Therefore $A^{\prime}, B^{\prime} \in \Delta\left(C_{n}\right)$ as desired.

\section{Algebraic properties of $t$-independence ideals of proper inter- val graphs}

For a graph $G$ on the vertex set $[n]$, the $t$-independence ideal of $G$, denoted by $I_{t}(G)$, is defined to be the ideal generated by all monomials $u=x_{i_{1}} x_{i_{2}} \cdots x_{i_{t}}$ for which $\left\{i_{1}, i_{2}, \ldots, i_{t}\right\}$ is a $(t-1)$-face of $\Delta(G)$. The $t$-independence ideal of $G$ is in fact the $t$-clique ideal of $G^{c}$. The class of $t$-clique ideals was introduced by Moradi [13] and had been further studied in [10] and [14]. In this section we consider the $t$-independence ideal of proper interval graphs and show that they have some nice algebraic properties.

The following result, which we quote from [3], will be of crucial importance in what follows. Let $I$ be a sortable monomial ideal, $A=K[u: u \in \mathcal{G}(I)]$ and $T=K\left[y_{u}: u \in \mathcal{G}(I)\right]$ be the polynomial ring over the field $K$ in the variables $y_{u}$ with $u \in \mathcal{G}(I)$. We let $L$ be the kernel of the $K$-algebra homomorphism from $T$ to $A$ with $y_{u} \mapsto u$ for $u \in \mathcal{G}(I)$.

Notice that if $(u, v)$ is an unsorted pair and $\left(u^{\prime}, v^{\prime}\right)=\operatorname{sort}(u, v)$, then $y_{u} y_{v}-y_{u^{\prime}} y_{v^{\prime}} \in L$ and $y_{u} y_{v}-y_{u^{\prime}} y_{v^{\prime}} \neq 0$, unless $\left(u^{\prime}, v^{\prime}\right)=(v, u)$. Relations of this form are called sorting relations. 
Theorem 12. There exists a monomial order $<^{\prime}$ on $T$, called sorting order, such that for each non-zero sorting relation $y_{u} y_{v}-y_{u^{\prime}} y_{v^{\prime}}$ the monomial $y_{u} y_{v}$ for the unsorted pair $(u, v)$ is the leading term. Moreover, the set of sorting relations forms a Gröbner basis of $L$.

Corollary 13. Let $\Delta$ be a t-sortable simplicial complex. Then the toric ring $K\left[x^{F}: F \in\right.$ $\Delta,|F|=t]$ is Koszul and a normal Cohen-Macaulay domain. In particular, when $G$ is a proper interval graph or an n-cycle, then $K\left[u: u \in \mathcal{G}\left(I_{t}(G)\right)\right]$ is Koszul and a normal Cohen-Macaulay domain.

Proof. Since the ideal $I=\left\langle x^{F}: F \in \Delta,|F|=t\right\rangle$ is sortable, by Theorem 12, the defining ideal $L$ of $A=K[u: u \in \mathcal{G}(I)]$ has a quadratic Gröbner basis with respect to the sorting order. It follows that $A$ is Koszul, see for example [6, Theorem 2.28]. Since the initial ideal of $L$ is squarefree, by the theorem of Sturmfels [16] (see also [6, Corollary 4.26]), $A$ is normal. Now we apply the result of Hochster [2, Theorem 6.3.5] which says that any normal toric ring is Cohen-Macaulay. The second statement is obtained by applying the first part on $\Delta=\Delta(G)$, Theorem 8, Remark 3(ii) and Proposition 11.

We now consider the Rees ring of the $t$-independence ideals of proper interval graphs. To this end we recall the concept introduced in [7], which is called the $\ell$-exchange property.

Let $I \subset S=K\left[x_{1}, \ldots, x_{n}\right]$ be a monomial ideal generated in a single degree. Then $A=K[u: u \in \mathcal{G}(I)]$ is isomorphic to the fiber $\mathcal{R}(I) / \mathfrak{m} \mathcal{R}(I)$ of the Rees $\operatorname{ring} \mathcal{R}(I)=$ $\bigoplus_{k \geqslant 0} I^{k} t^{k}$, where $\mathfrak{m}=\left\langle x_{1}, \ldots, x_{n}\right\rangle$ is the graded maximal ideal of $S$. Then $\mathcal{R}(I) \cong R / J$, where $R=S\left[y_{u}: u \in \mathcal{G}(I)\right]$ and $J$ is the kernel of the $K$-algebra homomorphism $R \rightarrow \mathcal{R}(I)$ with $x_{i} \mapsto x_{i}$ for $i=1, \ldots, n$ and $y_{u} \mapsto u t$ for any $u \in \mathcal{G}(I)$.

Let $A, T$ and $L$ be defined as before Theorem 12. We fix a monomial order $<^{\prime}$ on $T$. A monomial $w \in T$ is called a standard monomial of $L$ with respect to $<^{\prime}$, if $w \notin$ in $_{<^{\prime}}(L)$.

For example, if $I$ is sortable and we let $<^{\prime}$ be the sorting order on $T$, then $w=$ $y_{u_{1}} \cdots y_{u_{N}}$ is a standard monomial of $L$ with respect to $<^{\prime}$ if and only if $\left(u_{i}, u_{j}\right)$ is sorted for all $i<j$.

Definition 14. Let $I$ be a monomial ideal. Then $I$ is said to satisfy the $\ell$-exchange property with respect to the monomial order $<$ on $T$, if the following condition is satisfied: let $y_{u_{1}} \cdots y_{u_{N}}$ and $y_{v_{1}} \cdots y_{v_{N}}$ be any two standard monomials of $L$ with respect to $<$ such that

(i) $\operatorname{deg}_{x_{r}}\left(u_{1} \cdots u_{N}\right)=\operatorname{deg}_{x_{r}}\left(v_{1} \cdots v_{N}\right)$ for $r=1, \ldots, q-1$ with $q \leqslant n-1$,

(ii) $\operatorname{deg}_{x_{q}}\left(u_{1} \cdots u_{N}\right)<\operatorname{deg}_{x_{q}}\left(v_{1} \cdots v_{N}\right)$.

Then there exists an integer $k$, and an integer $q<j \leqslant n$ with $x_{j} \in \operatorname{supp}\left(u_{k}\right)$ such that $x_{q} u_{k} / x_{j} \in I$.

Proposition 3.1. Let $G$ be a proper interval graph on the vertex set $[n]$. Then for all $t \geqslant 2$, the ideal $I_{t}(G)$ satisfies the $\ell$-exchange property with respect to the sorting order. 
Proof. Let $y_{u_{1}} \cdots y_{u_{N}}$ and $y_{v_{1}} \cdots y_{v_{N}}$ be standard monomials satisfying (i) and (ii) of Definition 14. Then $\left(u_{i}, u_{j}\right)$ and $\left(v_{i}, v_{j}\right)$ are sorted for any $i<j$. If $u_{j}=x_{i_{j, 1}} \cdots x_{i_{j, t}}$ and $v_{j}=x_{i_{j, 1}^{\prime}} \cdots x_{i_{j, t}^{\prime}}$ for any $1 \leqslant j \leqslant N$, then by $[3$, Relation (6.3)],

$$
i_{1,1} \leqslant i_{2,1} \leqslant \cdots \leqslant i_{N, 1} \leqslant i_{1,2} \leqslant i_{2,2} \leqslant \cdots \leqslant i_{N, 2} \leqslant \cdots \leqslant i_{1, t} \leqslant i_{2, t} \leqslant \cdots \leqslant i_{N, t}
$$

and

$$
i_{1,1}^{\prime} \leqslant i_{2,1}^{\prime} \leqslant \cdots \leqslant i_{N, 1}^{\prime} \leqslant i_{1,2}^{\prime} \leqslant i_{2,2}^{\prime} \leqslant \cdots \leqslant i_{N, 2}^{\prime} \leqslant \cdots \leqslant i_{1, t}^{\prime} \leqslant i_{2, t}^{\prime} \leqslant \cdots \leqslant i_{N, t}^{\prime} .
$$

Since $\operatorname{deg}_{x_{r}}\left(u_{1} \cdots u_{N}\right)=\operatorname{deg}_{x_{r}}\left(v_{1} \cdots v_{N}\right)$ for $r=1, \ldots, q-1$ with $q \leqslant n-1$, it follows from the above sequences of inequalities that for any $i_{j, k} \leqslant q-1, i_{j, k}=i_{j, k}^{\prime}$. Hence $\operatorname{deg}_{x_{r}}\left(u_{j}\right)=\operatorname{deg}_{x_{r}}\left(v_{j}\right)$ for all $j$ and $1 \leqslant r \leqslant q-1$. Condition (ii) of Definition 14 implies that there exists $m$ such that $\operatorname{deg}_{x_{q}}\left(u_{m}\right)<\operatorname{deg}_{x_{q}}\left(v_{m}\right)$.

Let $u_{m}=x_{k_{1}} x_{k_{2}} \ldots x_{k_{t}}, v_{m}=x_{l_{1}} x_{l_{2}} \ldots x_{l_{t}}$ such that $k_{1}<\cdots<k_{t}$ and $l_{1}<\cdots<l_{t}$ and $q=l_{i}$ for some $1 \leqslant i<t$. Then $k_{1}=l_{1}, \ldots, k_{i-1}=l_{i-1}$ and $k_{i}>l_{i}=q$. Set $j=k_{i}$. We show that $x_{q} u_{m} / x_{j} \in I_{t}(G)$. By contradiction suppose that $\left(\operatorname{supp}\left(u_{m}\right) \backslash\left\{x_{j}\right\}\right) \cup\left\{x_{q}\right\}$ is not an independent set of $G$. Then $\left\{q, k_{r}\right\} \in E(G)$ for some $1 \leqslant r \leqslant t, r \neq i$. Since $k_{1}=l_{1}, \ldots, k_{i-1}=l_{i-1}$ and $\left\{x_{q}, x_{l_{1}}, \ldots, x_{l_{i-1}}\right\} \subseteq \operatorname{supp}\left(v_{m}\right)$, we have $r>i$. Hence $k_{r} \in N_{G^{q}}[q]$, where $G^{q}=G[q, q+1, \ldots, n]$. Observe that $q<k_{i}<k_{r}$ and $N_{G^{q}}[q]$ is an interval and a clique of $G$. This implies that $k_{i} \in N_{G^{q}}[q]$ and $\left\{k_{i}, k_{r}\right\} \in E(G)$. Since $\left\{x_{k_{i}}, x_{k_{r}}\right\} \subseteq \operatorname{supp}\left(u_{m}\right)$, it follows that $\operatorname{supp}\left(u_{m}\right)$ does not correspond to an independent set of $G$, a contradiction.

According to [7, Theorem 5.1] (see also [3, Theorem 6.24]), the Rees ring of a monomial ideal satisfying the $\ell$-exchange property has a particularly nice presentation. To describe this result, let $<_{\text {lex }}$ be the lexicographic order on $S$ with respect to $x_{1}>\cdots>x_{n}$. A new monomial order $<_{\text {lex }}^{\prime}$ on $R$ is defined as follows: for two monomials $u_{1}, u_{2} \in S$ and two monomials $v_{1}, v_{2} \in T$, we set $u_{1} v_{1}<_{\operatorname{lex}}^{\prime} u_{2} v_{2}$ if and only if (i) $u_{1}<_{\operatorname{lex}} u_{2}$ or (ii) $u_{1}=u_{2}$ and $v_{1}<v_{2}$.

Theorem 15. Let I be a monomial ideal generated in one degree, satisfying the $\ell$-exchange property. Then the reduced Gröbner basis of the toric ideal $J$ with respect to $<_{\text {lex }}^{\prime}$ consists of all binomials belonging to the reduced Gröbner basis of $L$ with respect to $<$ together with the binomials

$$
x_{i} y_{u}-x_{j} y_{v},
$$

where $x_{i}>x_{j}$ with $x_{i} u=x_{j} v$ and $j$ is the smallest integer for which $x_{i} u / x_{j}$ belongs to $I$.

Let $I \subset S=K\left[x_{1}, \ldots, x_{n}\right]$ a graded ideal and $P$ be a prime ideal with $I \subseteq P$. Recall that $I$ satisfies the strong persistence property with respect to $P$ if for all $k$ and all $f \in\left(\left(I S_{P}\right)^{k}: P S_{P}\right) \backslash\left(I S_{P}\right)^{k}$ there exists $g \in I S_{P}$ such that $f g \notin\left(I S_{P}\right)^{k+1}$. The ideal $I$ is said to satisfy the strong persistence property if it satisfies the strong persistence property with respect to $P$ for any prime ideal $P$ containing $I$. Note that strong persistence implies persistence, which means that $\operatorname{Ass}\left(I^{k}\right) \subseteq \operatorname{Ass}\left(I^{k+1}\right)$ for all $k$. 
It is shown in [8, Theorem 1.3] that $I$ satisfies strong persistence if and only if $I^{k+1}$ : $I=I^{k}$ for all $k$. Under the assumption that $K$ is infinite $I$ satisfies strong persistence if $\mathcal{R}(I)$ is normal or Cohen-Macaulay, see [8, Corollary 1.6].

As a result of Proposition 3.1 and Theorem 15 we obtain

Corollary 16. Let $G$ be a proper interval graph. Then for all $t \geqslant 2$, the independence ideal $I_{t}(G)$ satisfies the strong persistence property and all of its powers have linear resolutions.

Proof. It follows from Proposition 3.1 and Theorem 15 that all powers of $I_{t}(G)$ have linear resolution, see [5, Corollary 10.1.7]. By the results of Sturmfels and Hochster, mentioned already in the proof of Corollary 13, we see that $\mathcal{R}\left(I_{t}(G)\right)$ is a normal Cohen-Macaulay ring. By $[8$, Corollary 1.6], this implies strong persistence.

The following theorem which is a generalization of Theorem 2.4 in [10], proves conjecture 2.3 in [10] for proper interval graphs.

Theorem 17. Let $G$ be a proper interval graph on the vertex set $[n]$ and $I=I_{t}(G)$ for some $t \geqslant 2$. Then for any positive integer $m, I^{m}$ has linear quotients.

Proof. Note that

$$
I=\left\langle x_{i_{1}} \cdots x_{i_{t}}:\left\{i_{1}, \ldots, i_{t}\right\} \in S_{t}(G)\right\rangle,
$$

where $S_{t}(G)$ is the set of all $t$-independent subsets of $G$. Firstly we establish

$$
\begin{gathered}
\mathcal{A}=\left\{x_{i_{1,1}} x_{i_{1,2}} \cdots x_{i_{1, m}} x_{i_{2,1}} x_{i_{2,2}} \cdots x_{i_{2, m}} \cdots x_{i_{t, 1}} x_{i_{t, 2}} \cdots x_{i_{t, m}}:\right. \\
i_{1,1} \leqslant \cdots \leqslant i_{1, m} \leqslant i_{2,1} \leqslant \cdots \leqslant i_{2, m} \leqslant \cdots \leqslant i_{t, 1} \leqslant \cdots \leqslant i_{t, m}, \text { and } \\
\left.\left\{i_{1, \ell}, i_{2, \ell}, \ldots, i_{t, \ell}\right\} \in S_{t}(G) \text { for all } 1 \leqslant \ell \leqslant m\right\},
\end{gathered}
$$

is a minimal set of monomial generators for $I^{m}$. To this aim, note that any minimal monomial generator $u$ of $I^{m}$ is the product of $m$ monomials corresponding to some members of $S_{t}(G)$ and so is of degree $t m$. Thus it can be written as

$$
u=x_{i_{1,1}} x_{i_{1,2}} \cdots x_{i_{1, m}} x_{i_{2,1}} x_{i_{2,2}} \cdots x_{i_{2, m}} \cdots x_{i_{t, 1}} x_{i_{t, 2}} \cdots x_{i_{t, m}},
$$

where $i_{1,1} \leqslant \cdots \leqslant i_{1, m} \leqslant i_{2,1} \leqslant \cdots \leqslant i_{2, m} \leqslant \cdots \leqslant i_{t, 1} \leqslant \cdots \leqslant i_{t, m}$. Assume, in contrary, that there exists an index $1 \leqslant \ell \leqslant m$ such that $\left\{i_{1, \ell}, i_{2, \ell}, \ldots, i_{t, \ell}\right\} \notin S_{t}(G)$. Then there exist $1 \leqslant j<j^{\prime} \leqslant t$ such that either $i_{j, \ell}=i_{j^{\prime}, \ell}$ or $\left\{i_{j, \ell}, i_{j^{\prime}, \ell}\right\} \in E(G)$. Consider the multiset $C=\left\{i_{j, \ell}, i_{j, \ell+1}, \ldots, i_{j, m}, \ldots, i_{j^{\prime}, 1}, i_{j^{\prime}, 2}, \ldots, i_{j^{\prime}, \ell}\right\}$. Since $G$ is proper interval, any two vertices in $C$ are either equal or adjacent in $G$. Let $u_{1}, \ldots, u_{m} \in \mathcal{G}(I)$ such that $u=\prod_{i=1}^{m} u_{i}$. Since $\mathbf{x}^{C} \mid \prod_{i=1}^{m} u_{i}$ and $\operatorname{deg}\left(\mathbf{x}^{C}\right) \geqslant m+1$, there exists some $1 \leqslant i \leqslant m$ and $p, q \in C$ such that $x_{p} x_{q} \mid u_{i}$. Note that $u_{i}$ is squarefree and hence $p$ and $q$ are distinct. Since $\operatorname{supp}\left(u_{i}\right)$ corresponds to an independent set of $G$, we should have $\{p, q\} \notin E(G)$. This is a contradiction.

Conversely, it is obvious that any member of $\mathcal{A}$ is the product of $m$ monomials corresponding to members of $S_{t}(G)$ and so belongs to $I^{m}$. 
Now consider the lex order induced by $x_{1}>x_{2}>\cdots>x_{n}$ on the minimal monomial generators of $I^{m}$. Let $u, u^{\prime} \in \mathcal{A}$ with $u^{\prime}>_{\text {lex }} u$. Let

$$
u=x_{i_{1,1}} x_{i_{1,2}} \cdots x_{i_{1, m}} x_{i_{2,1}} x_{i_{2,2}} \cdots x_{i_{2, m}} \cdots x_{i_{t, 1}} x_{i_{t, 2}} \cdots x_{i_{t, m}}
$$

and

$$
u^{\prime}=x_{i_{1,1}^{\prime}} x_{i_{1,2}^{\prime}} \cdots x_{i_{1, m}^{\prime}} x_{i_{2,1}^{\prime}} x_{i_{2,2}^{\prime}} \cdots x_{i_{2, m}^{\prime}} \cdots x_{i_{t, 1}^{\prime}} x_{i_{t, 2}^{\prime}} \cdots x_{i_{t, m}^{\prime}} ;
$$

such that $i_{1,1} \leqslant i_{1,2} \leqslant \cdots \leqslant i_{1, m} \leqslant i_{2,1} \leqslant \cdots \leqslant i_{2, m} \leqslant \cdots \leqslant i_{t, 1} \leqslant \cdots \leqslant i_{t, m}$ and $i_{1,1}^{\prime} \leqslant i_{1,2}^{\prime} \leqslant \cdots \leqslant i_{1, m}^{\prime} \leqslant i_{2,1}^{\prime} \leqslant \cdots \leqslant i_{2, m}^{\prime} \leqslant \cdots \leqslant i_{t, 1}^{\prime} \leqslant \cdots \leqslant i_{t, m}^{\prime}$ and $\left\{i_{1, \ell}, i_{2, \ell}, \ldots, i_{t, \ell}\right\} \in$ $S_{t}(G)$ and $\left\{i_{1, \ell}^{\prime}, i_{2, \ell}^{\prime}, \ldots, i_{t, \ell}^{\prime}\right\} \in S_{t}(G)$ for all $1 \leqslant \ell \leqslant m$. Let $i_{s, k}$ be the smallest index such that $i_{s, k} \neq i_{s, k}^{\prime}$ for some $1 \leqslant s \leqslant t$ and $1 \leqslant k \leqslant m$. Then for any $1 \leqslant s^{\prime}<s$ and any $1 \leqslant k^{\prime} \leqslant m$, one has $i_{s^{\prime}, k^{\prime}}=i_{s^{\prime}, k^{\prime}}^{\prime}$ and for any $1 \leqslant k^{\prime}<k, i_{s, k^{\prime}}=i_{s, k^{\prime}}^{\prime}$ and $i_{s, k}^{\prime}<i_{s, k}$. So, if we set $u^{\prime \prime}=\frac{x_{i_{s, k}^{\prime}}}{x_{i_{s, k}}} u$, we have $u^{\prime \prime}>_{\text {lex }} u, u^{\prime \prime}: u=x_{i_{s, k}^{\prime}}$ and $x_{i_{s, k}^{\prime}} \mid u^{\prime}: u$. Hence it remains to prove that $u^{\prime \prime} \in \mathcal{A}$. Since $u \in \mathcal{A}$, it is sufficient to show that $S=\left\{i_{1, k}, i_{2, k}, \ldots, i_{s-1, k}, i_{s, k}^{\prime}, i_{s+1, k}, \ldots, i_{t, k}\right\} \in S_{t}(G)$. Suppose in contrary that $S$ is not an independent set of vertices of $G$. Since $u, u^{\prime} \in \mathcal{A}$, there is an integer $s+1 \leqslant j \leqslant t$ such that $\left\{i_{s, k}^{\prime}, i_{j, k}\right\} \in E(G)$. Since $i_{s, k}^{\prime}<i_{s, k}<i_{j, k}$ and $G$ is proper interval, $\left\{i_{s, k}, i_{j, k}\right\} \in E(G)$ which contradicts to $u \in \mathcal{A}$. So, we have $S \in S_{t}(G)$ which implies that $u^{\prime \prime} \in \mathcal{A}$ as desired.

\section{References}

[1] K. P. Bogart and D. West. A short proof that 'proper = unit'. Discrete Math., 201(1):21-23, 1999.

[2] W. Bruns and J. Herzog. Cohen-Macaulay rings. Cambridge Studies in advanced mathematics 39, Cambridge University Press, Cambridge, UK, 1998.

[3] V. Ene and J. Herzog. Gröbner bases in commutative algebra. American Mathematical Society, 2011.

[4] F. Gardi. The Roberts characterization of proper and unit interval graphs. Discrete Math., 307(22):2906-2908, 2007.

[5] J. Herzog and T. Hibi. Monomial ideals. Graduate Texts in Mathematics. Springer, New York, 2010.

[6] J. Herzog, T. Hibi and H. Ohsugi. Binomial ideals. Graduate Texts in Mathematics. Springer, New York, 2018.

[7] J. Herzog, T. Hibi and M. Vladoiu. Ideals of fiber type and polymatroids. Osaka J. Math., 42:807-829, 2005.

[8] J. Herzog and A. Qureshi. Persistence and stability properties of powers of ideals. J. Pure Appl. Algebra, 219:530-542, 2015.

[9] J. Herzog, A. Rauf and M. Vladoiu. The stable set of associated prime ideals of a polymatroidal ideal. J. Algebraic Combin., 37(2):289-312, 2013. 
[10] F. Khosh-Ahang and S. Moradi. Some algebraic properties of t-clique ideals. Comm. Algebra, 47:2870-2882, 2019.

[11] P. J. Looges and S. Olariu. Optimal greedy algorithms for indifference graphs. Comput. Math. Appl., 25:15-25, 1993.

[12] J. Martinez-Bernal, S. Morey and R. H. Villarreal. Associated primes of powers of edge ideals. Collect. Math., 63(3):361-374, 2012.

[13] S. Moradi. $t$-clique ideal and $t$-independence ideal of a graph. Comm. Algebra, 46:3377-3387, 2018.

[14] S. Moradi, M. Rahimbeigi, F. Khosh-Ahang and A. Soleyman Jahan. A family of monomial ideals with the persistence property. J. Algebra Appl., 18(5):1950093, 2019.

[15] F. S. Roberts. Representations of indifference relations. Ph.D. Thesis, Stanford University, Stanford, CA, 1968.

[16] B. Sturmfels. Gröbner bases and convex polytopes. American Mathematical Society, 1996. 